\title{
The determination and evaluation of Nitinol constitutive models for finite element analysis
}

\author{
E. McCummiskey, a, W. M. Dempster ${ }^{1, \text { b }}$, D. H. Nash ${ }^{1, \mathrm{c}}$, T. R. \\ Ashton $^{2, \mathrm{~d}}$, D. G. Stevenson ${ }^{2, \mathrm{e}}$ \\ ${ }^{1}$ Dept. of Mechanical Engineering, University of Strathclyde, Glasgow, Scotland. G1 \\ $1 \mathrm{XJ}$ \\ ${ }^{2}$ Terumo Vascutek, Inchinnan, Scotland. PA24 9RR \\ aemma.mccummiskey@strath.ac.uk, bwilliam.dempster@strath.ac.uk, \\ cd.nash@strath.ac.uk, ${ }^{\mathrm{d}} \mathrm{t}$. ashton@vascutek.com, ${ }^{\mathrm{e}} \mathrm{d} . \mathrm{stevenson@vascutek.com}$
}

Keywords: Nitinol, fine wire, testing, shape memory alloy, strain rate, temperature dependency, constitutive model.

\begin{abstract}
Superelastic Ni-Ti (Nitinol) is a member of the shape memory alloy (SMA) family of metals. The physical properties of Nitinol are highly dependant on a number of factors, including manufacturing method, subsequent processing, operating temperature, and strain rate. These factors complicate the prescription of material constitutive models, leading to complexities in the computational analysis of Nitinol components. The current work explores the limitations in the Nitinol material model available in existing commercial finite element (FE) software using a series of specially design experimental tests and representative FE models.
\end{abstract}

\section{Introduction}

Superelastic Ni-Ti (Nitinol) a member of the shape memory alloy (SMA) family, has earned recognition in recent years for its attractive material properties such as its thermal memory, the recoverability of an original shape upon the application of heat subsequent to deformation at low temperature, and superelasticity, the existence of a large non-linearly recoverable strain upon loading and unloading[1]. This combined with the material's biocompatibility[2] has lead to Nitinol becoming the material of choice for many biomedical applications, including but not limited to cardiovascular stents, endovascular devices and orthodontic archwires. Devices prove difficult to test in 'real life' situations and so gainful insight into the working of the device can be gaining using computational modelling.

Material characterisation of Nitinol is difficult due to its sensitivity to the production process, temperature dependence, strain rate dependence, asymmetry in tension and compression and variation of response under cyclic loading which are reported in the literature[3-10]. If a realistic model of the material is to be obtained, then testing must be undertaken on truly representative samples. Nitinol is considered to be a superelastic material with recoverable strains of up to $8 \%$ [1] however this is not always the case, and a residual strain of up to $\sim 1 \%$ at higher temperatures is recorded. Variance is also observed on load cycling [7,9]. While some finite element packages do provide a built-in SMA material model[11-13] based on the work of Auricchio et al[14], the model does not fully incorporate the above behavioural traits of Nitinol which have been reported since the publication of the model. Hence it is the purpose of this paper to report on an evaluation of the Auricchio et al model [14] $]^{1}$ as 
implemented in the ANSYS finite element (FE) code with particular emphasis on cyclic and temperature effects.

The present work discusses full characterisation of $0.28 \mathrm{~mm}$ diameter Nitinol wire through testing and for subsequent use in the computational design of medical devices. The accuracy of the material constitutive model is examined by comparison of ANSYS (FE) simulations of the test conditions. As the material model available in ANSYS is typical of the majority of commercially available codes a more general statement can be made regarding the efficacy of these codes.

\section{Experimental Programme}

A test programme for Nitinol wire was devised to ascertain dependency on low magnitude cycling, temperature, and stress-strain asymmetry. For each test condition three specimens were considered to allow for testing errors. In addition to this the authors have also considered a wider sample range to investigate variation found in testing, however this is outside the scope of the current work.

Variation with low magnitude cyclic loading. Tensile testing of the $0.28 \mathrm{~mm}$ SE-508 $(55.6 \mathrm{wt} . \% \mathrm{Ni})$ wire with an Austenite finishing (Af) temperature of $11.75^{\circ} \mathrm{C}$ was carried out on a Tinius Olsen H5KS tensile tester using a gauge length of $200 \mathrm{~mm}$. Testing was temperature controlled at a temperature of $\mathrm{Af}+10^{\circ} \mathrm{C}$ to ensure no secondary effects. The material was cycled between $6 \%$ and $0.01 \%$ strain for 100 cycles at a frequency of $0.05 \mathrm{~Hz}$.

The variance in response is shown in Fig. 1 below where variation in the first linear modulus, shortening and depreciation of the upper plateau magnitude and the increase in residual strain can be seen. To track this change more clearly, the variation of the first linear modulus and residual strain are plotted from cycles 1 to 100 in Fig. 2.

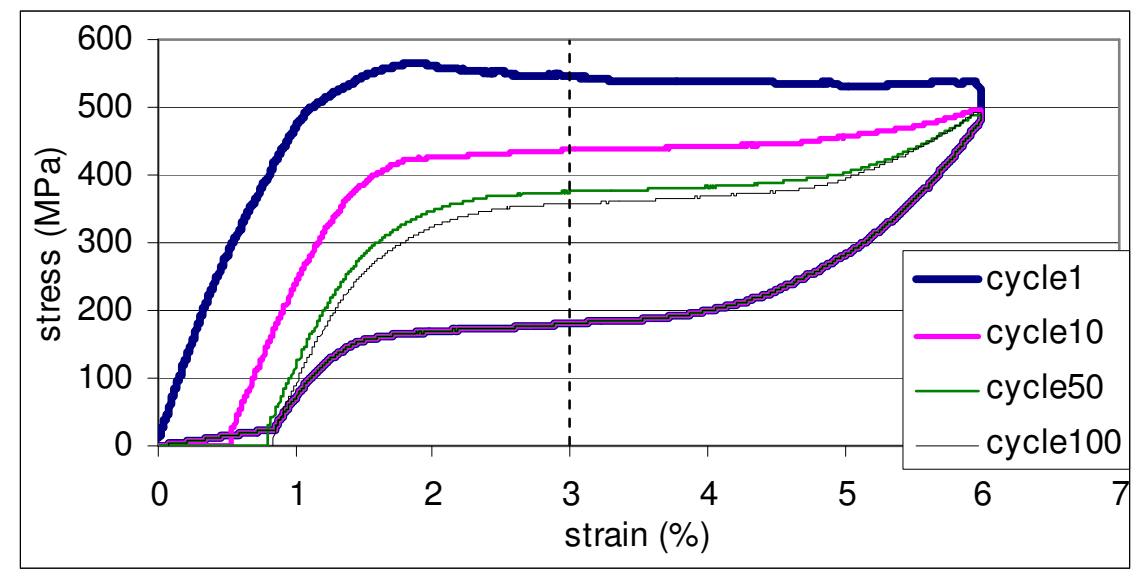

Fig. 1 Variation in Nitinol under cyclic loading for up to 100 cycles, and indicating the variation in loading plateau level at $3 \%$ strain.

\footnotetext{
${ }^{1}$ It should be noted that the work used by ANSYS to model shape memory alloy material is not the most recent work by Auricchio et al in this field and subsequent work has not been introduced into the ANSYS model.
} 

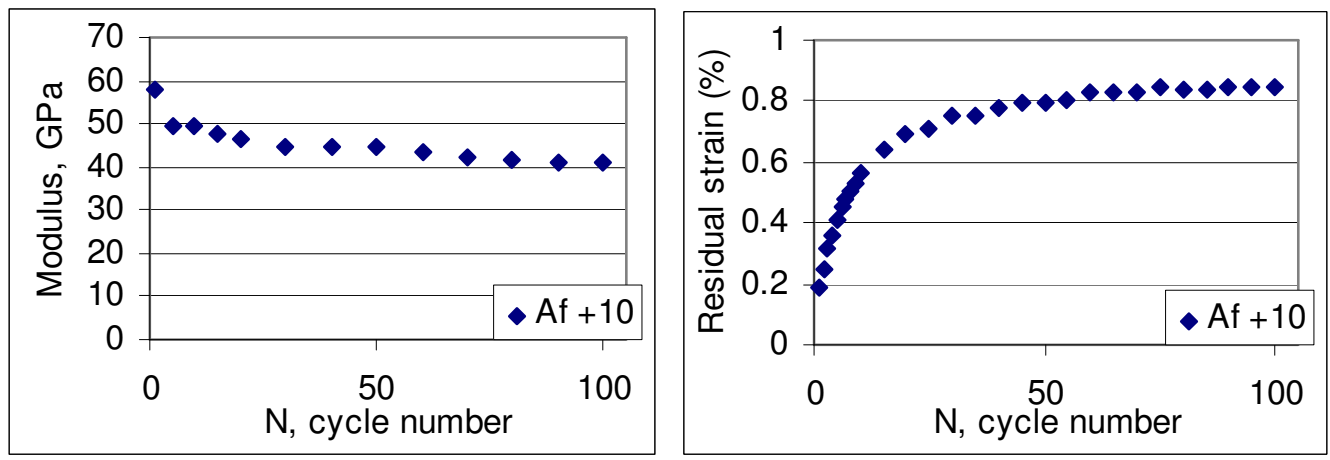

Fig. 2a) Variation of first linear modulus with cycling, and b) Variation in residual strain with cycling.

From Fig. 1 it is seen that through cycling, the start of the phase transformation from the material's parent phase into martensite occurs at a progressively higher strain level. It is suggested by Morgan et al [9] that this is due to small precipitates of martensite which remain in the stable austenite material after the first cycle.

The first linear modulus of the tested Nitinol (Fig. 1) decreases with cycling, with the proportion of reduction reducing each time, until the material remains largely stable after 20-30 loading cycles. The variation in residual strain, shown in Fig. 2 stabilises by 30-40 loading cycles at a value of $0.85 \% \varepsilon$ after 100 cycles. The computational model calls for a starting $\left(\sigma_{\mathrm{S}}^{\mathrm{SA}}\right)$ and finishing $\left(\sigma_{\mathrm{F}}^{\mathrm{SA}}\right)$ loading plateau stress, so it is beneficial to note that during the first cycle the $\sigma_{\mathrm{S}} \mathrm{SA}$ has a value of $566 \mathrm{MPa}$ at a strain of $1.89 \%$ and by the $100^{\text {th }}$ cycle a value of $353 \mathrm{MPa}$ occurring at a strain of $2.65 \%$, a reduction of over $37 \%$. The strain value increases by almost $30 \%$ due to the residuals strains discussed.

Variation with temperature. It is reported[4] that superelasticity has a limited region of effectiveness for a temperature window above the Af temperature, and that the superelasticity decreases with separation from Af. For any given application, variances in production and processing techniques could potentially change the operating temperature relative to Af and thus the response of the material. The $0.28 \mathrm{~mm}$ SE-508(55.6wt. \% Ni) wire was tested at temperatures of $\mathrm{Af}+10^{\circ} \mathrm{C}$ and $\mathrm{Af}+40^{\circ} \mathrm{C}$, under the same test conditions, loading to $6 \% \varepsilon$ with a gauge length of 200mm. The stress-strain curves obtained are shown in Fig. 3-a) which shows clearly the increase in first linear modulus, plateau stress, and in residual strain with increasing temperature. Fig.'s 1-3-a) demonstrated the variation in response through load cycling; an effect which may prevail at elevated temperatures. In order to investigate this, each test ran for 100 cycles and the stress strain responses at cycle 100 are compared in Fig. 3-b).

The data displayed for the $100^{\text {th }}$ cycle of the material shows an increasingly pronounced shift in the hysteresis curve with elevated temperature. At temperatures close to Af, the phase transformation of the material is clearly identifiable, however with an increased test temperature the hysteresis loop has degraded exhibiting a loading plateau modulus which is 7.5 times greater and an extra $1.005 \%$ of residual strain. 

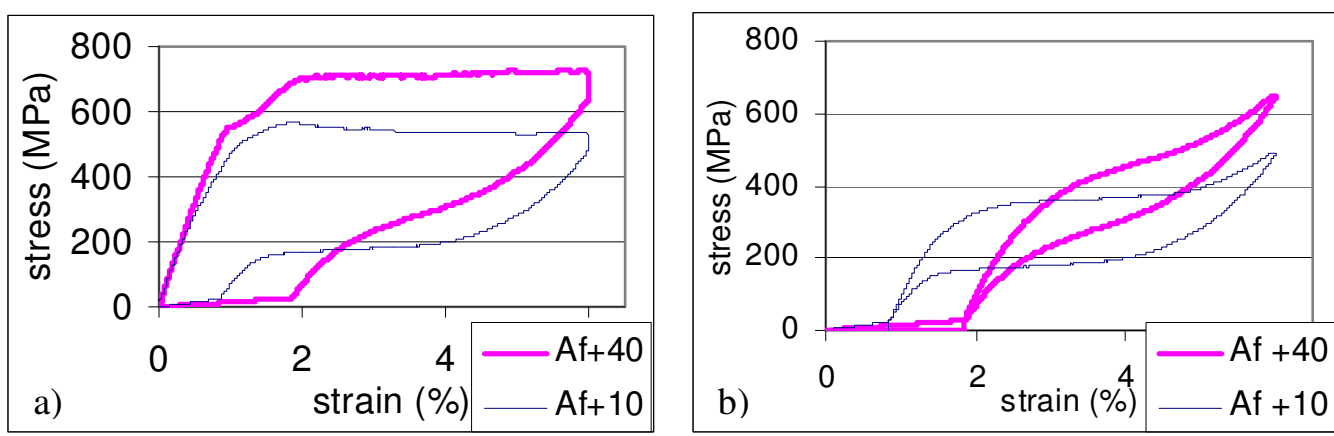

Fig. 3 Variation of stress-strain curve with temperature a) during first cycle, and b) after 100 loading cycles.

Asymmetry in tension and compression. Nitinol is known to display an asymmetrical response in tension and compression $[5,8,10]$ however it is very difficult to test fine diameter wire $(0.1-0.3 \mathrm{~mm}$ diameter) in compression and so an alternative technique must be employed.

In order to investigate this phenomenon, $1.8 \mathrm{~mm}$ diameter wire was sourced to the same specification as fine wire previously tested with an Af temperature of $-5.1^{\circ} \mathrm{C}$ and test temperature of $\mathrm{Af}+30^{\circ} \mathrm{C}$. The wire was tested in tension up to $6 \% \varepsilon$ and in compression up to $4 \% \varepsilon$ using a Zwick Rel 2061 testing machine to show asymmetry in tension and compression, see Fig. 4.

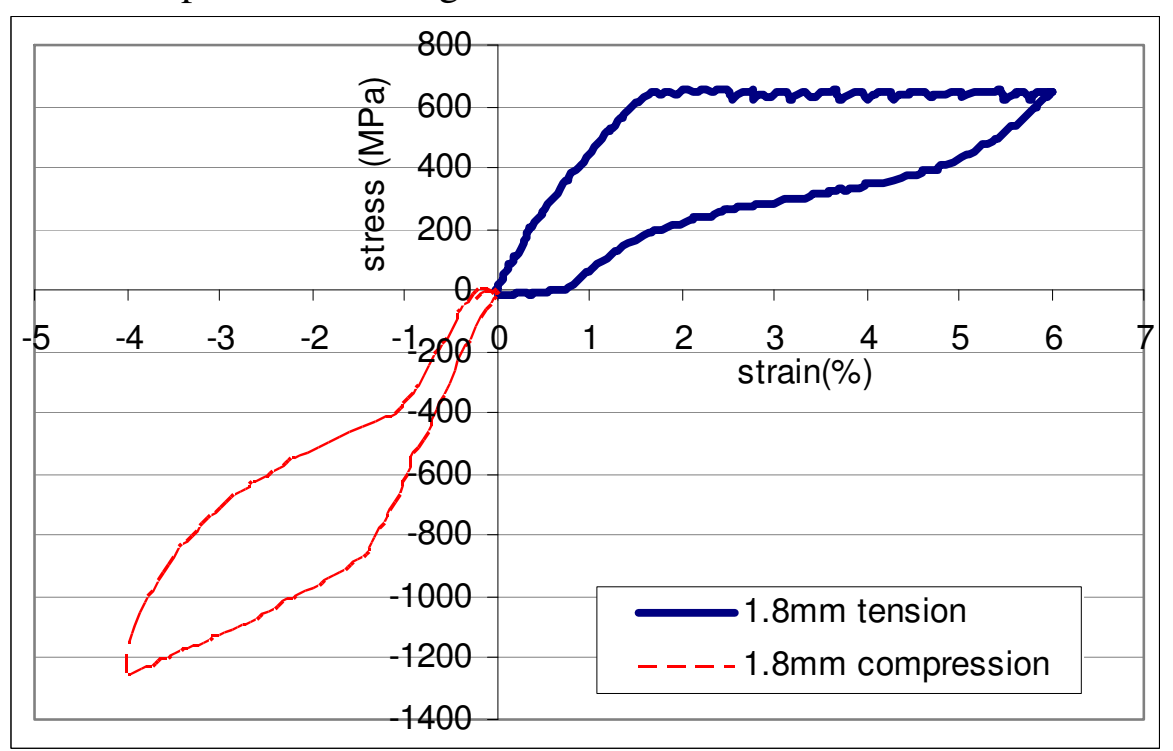

Fig. 4 Asymmetry of Nitinol in tension and compression.

The asymmetry of the Nitinol wire is evident in Fig. 4 above. The austenite starting stress $\left(\sigma_{\mathrm{S}}^{\mathrm{SA}}\right)$ is seen to be $645 \mathrm{MPa}$ in tension at a strain of $1.67 \%$, whilst in compression the value rises in magnitude to $853 \mathrm{MPa}$ at a strain of $-1.43 \%$ an increase of $24.3 \%$. The linear region modulus has a higher magnitude in compression and the plateau in compression is less pronounced even during the first cycle of the material. Testing limitations prohibit the current work from testing in compression above $4 \% \varepsilon$ however it is reported elsewhere[10] that a shorter plateau is also observed. 
The computational model evaluated the compression asymmetry using the austenite starting stress in compression $\left(\sigma_{\mathrm{S}}{ }^{\mathrm{SA}-}\right)$, which has been previously defined. However, this is a somewhat speculative value as the compression and tension tests have been completed on $1.8 \mathrm{~mm}$ diameter wire as opposed to the investigated value of $0.28 \mathrm{~mm}$.

Whilst the wires are sourced to the same specification, the high sensitivity of Nitinol to production methods and processing implies that there could be a significant difference in the responses of the two wires. Fig. 5 compares the tensile stress strain curve of the $1.8 \mathrm{~mm}$ wire with that of the $0.28 \mathrm{~mm}$ wire both tested at $\mathrm{Af}+30^{\circ} \mathrm{C}$ for comparison.

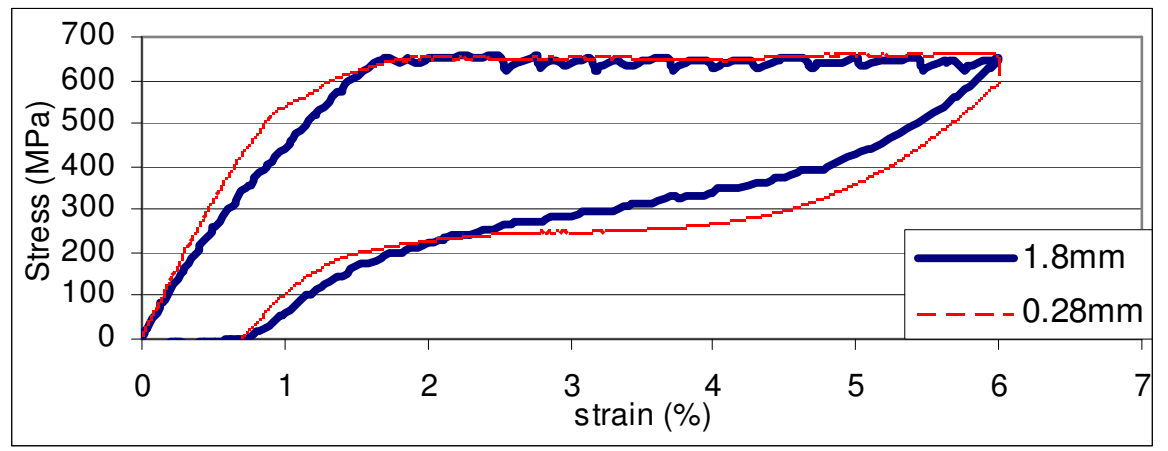

Fig. 5 Comparison of the $1.8 \mathrm{~mm}$ and $0.28 \mathrm{~mm}$ diameter wires in tension.

Both wires show similar stress-strain responses, with almost identical plateau levels, however the first linear modulus is higher for the finer wire as is the load-unload modulus seen at the end of the hysteresis loop. The overall values of residual strain compare very well. A contributing factor in the discrepancies of the response could be due to the change in testing machine and gripping method, required due to limitations of machines available. The $1.8 \mathrm{~mm}$ diameter wire data is therefore used as a basis for investigation into the computational model.

\section{Comparison with Computational Model}

The computational model available in commercial FE codes (e.g. ANSYS, ABAQUS, and LS-DYNA) spans from the work of Auricchio et al [14] which represents a simplified SMA model. This model does not include permanent strain, and represents the asymmetry in tension and compression with a single parameter which does not take into consideration the asymmetry seen in the first linear modulus or plateau length variation.

The present work shows that the material exhibits a variance in response through cycling and temperature. A computational model of the tensile test has been created which allows the limitations of the SMA constitutive model to be tested. This model consist of a wire of diameter $1.8 \mathrm{~mm}$ and length $10 \mathrm{~mm}$, which was then subjected to a longitudinal displacement of $0.6 \mathrm{~mm}$ at one end while the other end was fully fixed. The model consisted of 960 hexahedral elements and 4521 nodes. The structure is meshed with 3D structural solid elements of type 186 (ANSYS) which allow quadratic displacement behaviour and have three degrees of freedom per node. Fig. 6 below shows the geometry and element distribution of the model. 


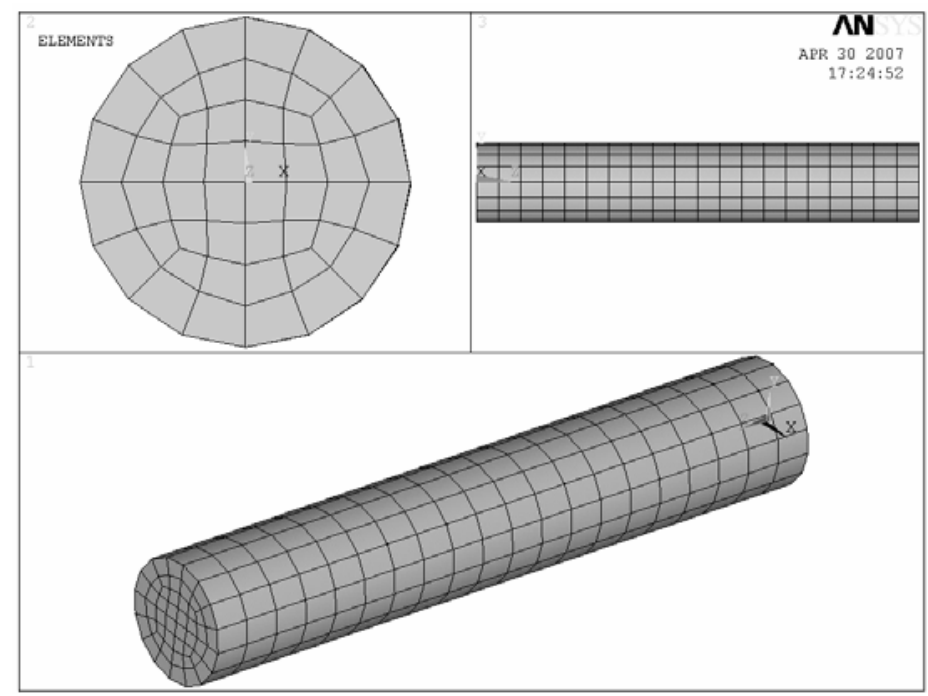

Fig. 6 FE model geometry and element distribution.

Variation with load cycling. The experimental data discussed previously was used as the input to the computational model created in ANSYS v10.0. Using data from the first cycle to define the model, a good correlation exists with the computational results in tension (Fig. 7a) though discrepancies exist at transitions to the plateaus, for the unloading portion of the curve and the permanent strain region. However if the computational model is put through 100 cycles as with the experimental data (Fig. $7 \mathrm{~b})$ the data no longer approximates the behaviour with the computational model remaining as the first cycle. This is also shown in Fig. 7c as the first 4 cycles are shown for the FE model which exhibit a constant response.
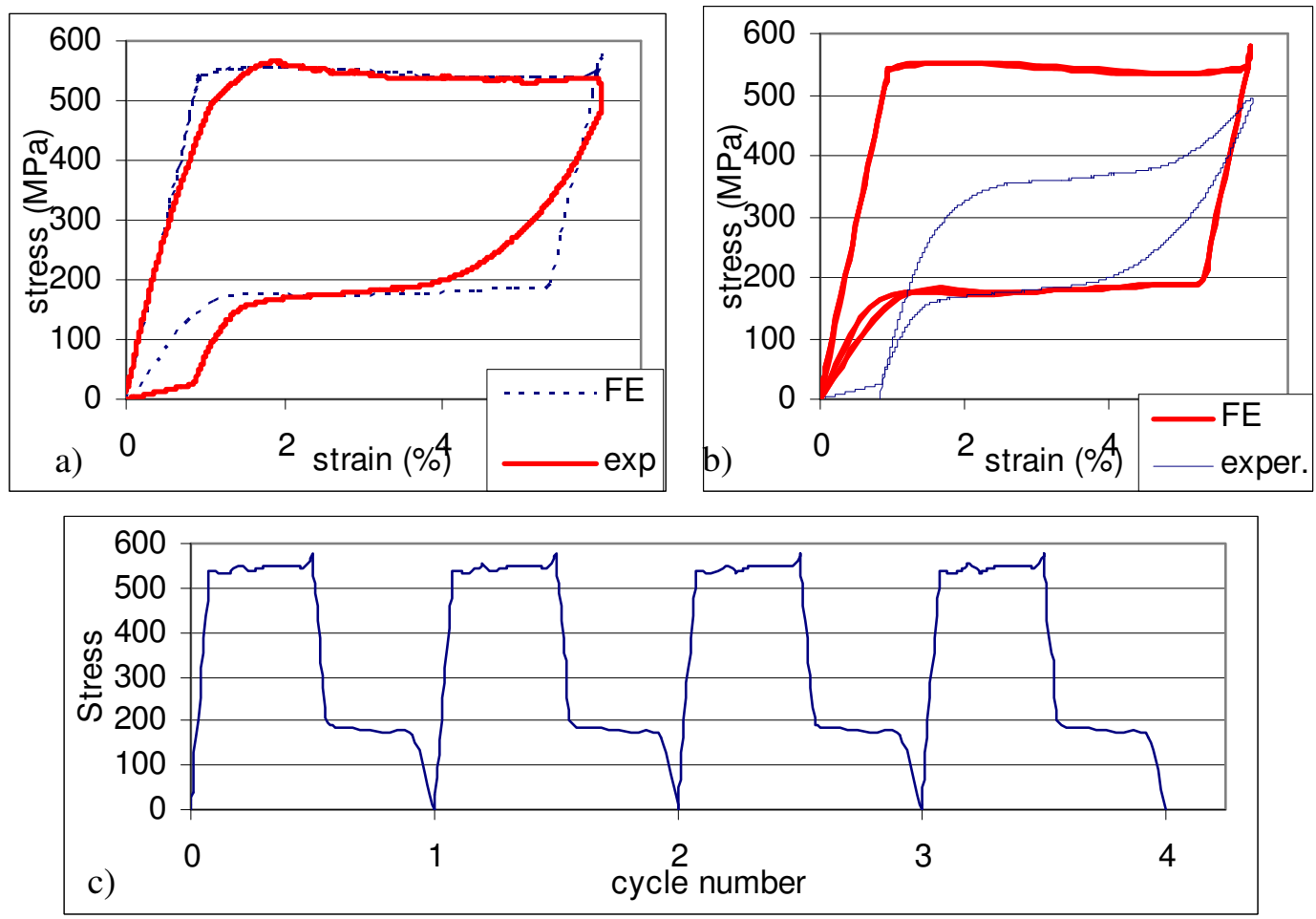

Fig. 7 a) Correlation between experimental and FE results for first cycle, b) Correlation of $100^{\text {th }}$ cycle data of experimental and FE results and c) showing loading response of subsequent cycles in FE. 
Variation with Temperature. Experimental data was input into the computational model at temperatures of $\mathrm{Af}+10^{\circ} \mathrm{C}$ and $\mathrm{Af}+40^{\circ} \mathrm{C}$ in order to set up temperature dependant material properties within the finite element model, where linear interpolation is used to determine properties at intermediate temperature conditions. Tensile tests were then simulated for the first loading cycle using ANSYS at a temperature of $\mathrm{Af}+20^{\circ} \mathrm{C}$ then $\mathrm{Af}+30^{\circ} \mathrm{C}$ to investigate how the constitutive model replicates temperature variation. The correlation of experimental to computational response is show in Fig. 8 below.
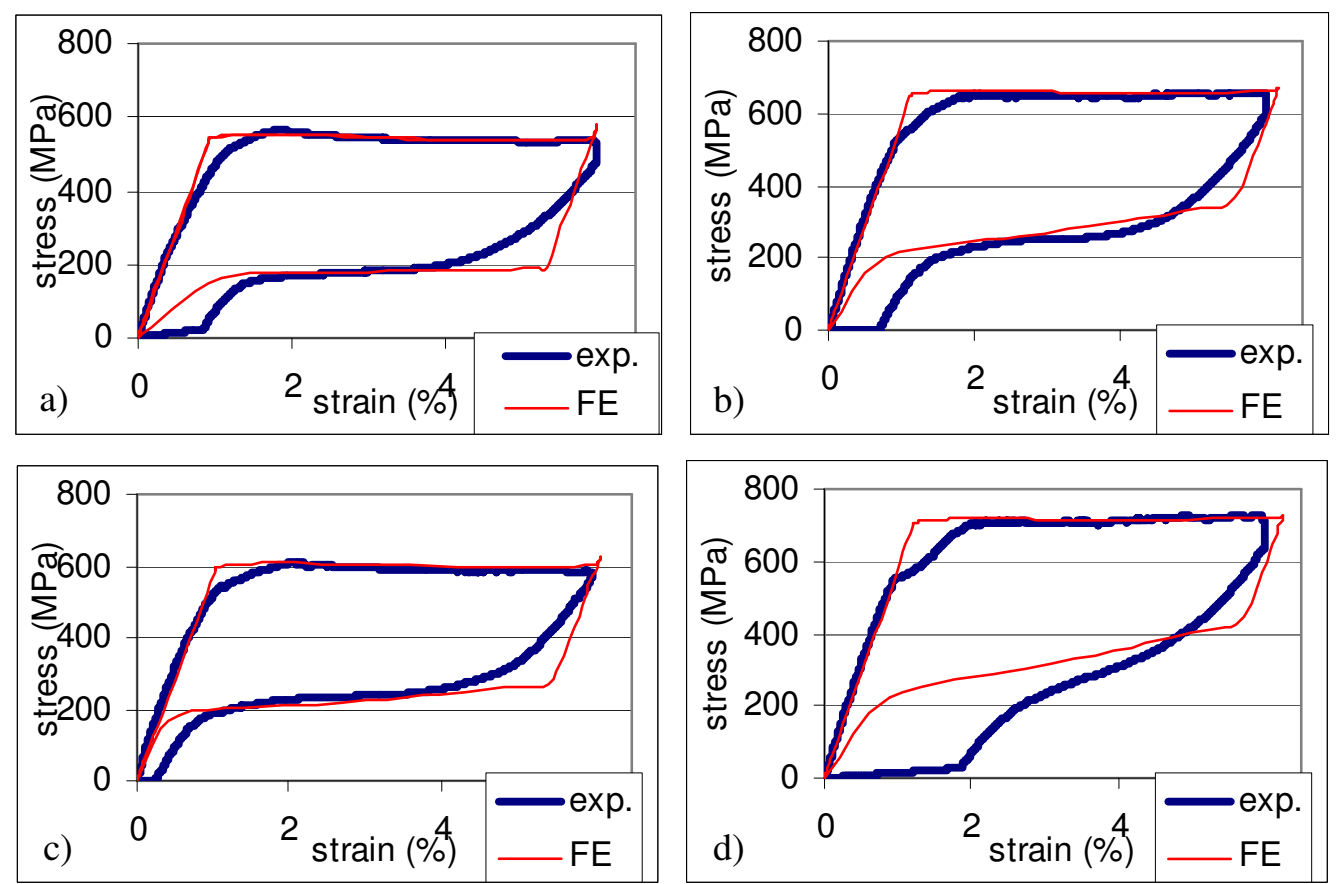

Fig. 8 Stress-strain variation in computational \& experimental material response for a) $\left.\left.\mathrm{Af}+10^{\circ} \mathrm{C}, \mathrm{b}\right) \mathrm{Af}+20^{\circ} \mathrm{C}, \mathrm{c}\right) \mathrm{Af}+30^{\circ} \mathrm{C}$ and d) $\mathrm{Af}+40^{\circ} \mathrm{C}$.

The computational material model responds well to changes in temperature due to the linear nature of the variation in material response seen with temperature for the first loading cycle of the material.

\section{Conclusions}

Results from an extensive material characterisation testing programme for Nitinol SE508 wire of diameter $0.28 \mathrm{~mm}$ have been presented. The issues associated with testing small diameter wire have been addressed and it is possible to test fine wire accurately in tension but more difficult in compression. Material characterisation in terms of a representative stress-strain curve has shown significant influence in the behaviour resulting from low amplitude cycling, temperature effects and tension and compression testing. An attempt to replicate the tension-compression asymmetry of the wire has also been made. The limitations of Auricchio et al's [14] 1997 model, used in modern commercial finite element packages was explored with specific interest given to the model's response after cycling and temperature variation.

The computational model performs well against the experimental data used, especially during the first linear elastic region and the loading plateau. The variation in modulus between the first and second linear region moduli is not represented in the model provided by ANSYS which explains the inaccuracy of the material model 
during the initial unload phase of the model, and the under representation of the permanent strain in the model leads to a distinct variance from the experimental data especially at elevated temperatures. The variance seen upon cycling is not represented by the computational model; however the temperature dependence of the material can be accurately depicted within the aforementioned bounds if material data for a high and low temperature can be given.

For the generic applications considered within the current work, namely medical devices, the model does not perform with an unreasonable remit of accuracy. For the medical device application examined by the authors, the device does not exist in the zero-stress state and thus the full unloading and permanent strain issues are for the large part inconsequential. For other applications, further consideration should be given to this on a case by case basis.

\section{References}

1. Otsuka, K., Wayman, C. M., (1998). Shape Memory Materials: Cambridge University Press.

2. Duerig, T., Pelton, A., Stockel,D., (1999). 'An Overview of nitinol medical applications'. Materials Science and Engineering A, A273-275:149-160.

3. Favier, D., Liu, Y., Orgeas, L., Sandel, A., Debove, L., Comte-Gaz, P., (2006). 'Influence of thermomechanical processing on the superelastic properties of a Ni-rich Nitinol shape memory alloy'. Materials Science and Engineering A, A429:130-136.

4. Otsuka, K., Ren, X., (2005). 'Physical Metallurgy of Ti-Ni-based shape memory alloys'. Progress in Materials Science, 50:511-678.

5. Adharapuapu, R., A.. Jiang, F., Vecchio. K., S., Gray., G., T., (2006). 'Response of NiTi shape memory alloy at high strain rate: A systematic investigation of temperature effects on tension-compression asymmetry'. Acta Materialia, 54:4609-4620.

6. Chen, W., W., Wu, Q., Kang, J., Winfree, N., (2008). 'Compressive superelastic behaviour of a NiTi shape memory alloy at strain rates of 0.001750s-1'. International Journal of Solids and Structures, 38:8989-8998.

7. Gong, J., M., Tobushi, H., Takaka, K., Okumara, K., (2002). 'Superelastic deformation of a TiNi shape memory alloy subjected to various cyclic loadings'. Proceedings of the Institution of Mechanical Engineers Part L: Journal of Material: Design and Applications, 216 (1):17-23.

8. Liu, Y., Xie, Z., Van Humbeeck, J., Delaey, L., (1998). 'Asymmetry of stressstrain curves under tension and compression for NiTi shape memory alloys'. Acta Materialia, 46:4325-4338.

9. Morgan, N., B., Gong. X.-Y., Wick, A., Pelton, A., Journal of ASTM International, 2006(IN PRINT). 'Observations on cyclic transformation behaviour of Nitinol'.

10. Siddons, D.J., Moon, J. R., (2001). 'Tensile and compression performance of NiTi tubing'. Materials Science and Technology, 17 (9):1073-1078.

11. ANSYS Inc., (2005). 'Release 10.0 Documentation for ANSYS'.

12. Hibbit, Karlsson \& Sorensen Inc., 'Abaqus User's Manual Version 6.5'.

13. LS-Dyna, (2006). 'LS-Dyna Theory Manual'.

14. Auricchio, F., Taylor, R. L., Lubliner, J., (1997). 'Shape-memory alloys: macromodelling and numerical simulations of the superelastic behaviour'. Computer Methods in Applied Mechanics and Engineering, 146:281-312. 